\title{
VINCULAÇÃO AOS PAIS E ANSIEDADE EM JOVENS ADULTOS
}

\author{
Maria da Graça Silva ${ }^{1}$ \\ Maria Emília Costa ${ }^{2}$
}

Resumo: Este estudo tem como principal objectivo analisar a relação entre os estilos de vinculação aos pais, baseados no modelo de Bartholomew e Horowitz (1991) e a ansiedade em jovens. Na presente investigação foi utilizada uma amostra de 511 jovens portugueses, com idades compreendidas entre os 17 e os 26 anos, que frequentavam diferentes cursos universitários em estabelecimentos de ensino superior da área metropolitana do Porto. De modo geral, verificam-se correlações significativas baixas da qualidade de laço emocional e inibição da exploração e individualidade à mãe e ao pai, com os medos e características obsessivo-compulsivas, e correlações significativas positivas baixas ou moderadamente baixas entre a dimensão ansiedade de separação e dependência à mãe e ao pai com a ansiedade. Relativamente aos estilos de vinculação, registam-se efeitos significativos nos vários tipos de medo e nos pensamentos obsessivos e comportamentos compulsivos. Verifica-se que os sujeitos com estilo de vinculação insegura assumem médias mais elevadas, na maior parte dos medos e nas características obsessivo-compulsivas. São ainda discutidas as implicações desta investigação para a intervenção psicológica junto dos jovens, no sentido de promover o desenvolvimento psicológico, particularmente uma visão positiva de si próprio e dos outros, e prevenir a estruturação da ansiedade.

Palavras-chave: vinculação, ansiedade, jovens adultos.

Attachment to parents and anxiety in young adults (Abstract): The main goal of this study was the analysis of the relationship between attachment to parents style, based in Bartholomew \& Horowitz (1991) and anxiety in young adults. The present research was developed in a sample of 511 portuguese youngsters, with ages between 17 and 26 years, which attended several higher education courses in the Porto area. In general, we have found significant low correlations between the quality of bonding and exploration and individuation inhibition to mother and father and fears and obsessive-compulsive characteristics. We also have found

\footnotetext{
${ }^{1}$ Mestre em Psicologia na Área de Consulta Psicológica de Jovens e Adultos; Técnica Superior da Faculdade de Psicologia e de Ciências da Educação da Universidade do Porto.

2 Professora Associada com Agregação da Faculdade de Psicologia e de Ciências da Educação da Universidade do Porto. Coordenadora do Instituto de Consulta Psicológica, Formação e Desenvolvimento da F.P.C.E. da Universidade do Porto.
} 
significant low positive correlations or moderately low correlations between the separation anxiety and dependency to mother and father and anxiety. With respect to attachment style, we have registered significant effects in the different kinds of fear and in the obsessive thoughts and compulsive behaviors: subjects with insecure attachment style have presented higher means in most of the fears and obsessive-compulsive characteristics. We discuss implications of results to psychological development, specifically a more positive view of themselves and of the others and to prevent anxiety structuration as well.

\section{Introdução}

É vasta a literatura que refere a origem multifactorial da ansiedade, sendo vários os estudos que enfatizam a importância das causas genéticas (Beidel, Turner \& Morris, 1999; Boer \& Lindhout, 2001), relacionadas com características temperamentais, como a inibição comportamental (Oosterlaan, 2001; Lonigan \& Phillips, 2001), ou causas familiares, como os estilos educativos e práticas de socialização (Morris, 2001). Mais recentemente tem-se valorizado abordagens teóricas desenvolvimentais para se perceber os percursos adaptativos ou desadaptativos que os indivíduos podem encontrar, ao longo das várias mudanças que vão decorrendo ao longo da sua vida. Com este propósito, a teoria da vinculação tem sido referida como modelo explicativo para o desenvolvimento e manutenção da ansiedade (Dadds \& Roth, 2001; Canavarro, 1999; Manassis, 2001).

Esta teoria postula que as crianças, por uma questão de sobrevivência, tendem a procurar proximidade do seu cuidador (geralmente, a mãe), e este tende a responder-lhe de modo complementar (Bowlby, 1973). Como resultado destas interacções, desenvolve-se um laço afectivo entre a criança e o cuidador que os une e perdura no tempo, designado por vinculação (Ainsworth, 1989; Bowlby, 1979). As crianças com diferentes tipos de vinculação desenvolvem cognições diferentes relativamente às relações interpessoais e diferem no modo como regulam o seu afecto. Bowlby (1982) designou as representações mentais do self, da figura de vinculação, de outras relações significativas e do mundo, por modelos representacionais ou modelos internos dinâmicos (internal working model). É a existência destes modelos mentais que explica como é que a interacção com as pessoas significativas pode influenciar o desenvolvimento emocional e social.

Bowlby $(1973,1980)$ sugere que, quando a criança desenvolve representações negativas acerca de si própria e dos outros, está mais vulnerável para desenvolver perturbações psicológicas. Este autor explica o desenvolvimento da ansiedade através de padrões relacionais com as figuras de vinculação centrados sobretudo em rejeição ou superproteção. 
Quando as figuras de vinculação são responsivas e acessíveis, as crianças desenvolvem um modelo de si próprias, como ser amado, estimado e respeitado, e um modelo dos outros como pessoas sensíveis, confiáveis e carinhosas (Bretherton, 1985). Esta confiança em si própria e nos outros leva a criança a desenvolver estratégias seguras de procura da figura de vinculação em situações de perigo. Quando, pelo contrário, as figuras de vinculação são indisponíveis, inconsistentes ou rejeitantes, as crianças desenvolvem um modelo si como não sendo dignas de amor ou não tendo valor e, simultaneamente, um modelo dos outros como insensíveis e rejeitantes. Estas crianças, como não confiam na disponibilidade da figura de vinculação, tendem a ter o seu sistema de vinculação cronicamente activado, mesmo em situações com perigo diminuto, e a diminuir a sua exploração (Manassis, 2001).

Recentemente, Bartholomew e Horowitz (1991) retomam o conceito de modelos internos dinâmicos de Bowlby e propõem um modelo bidimensional de vinculação, baseado na suposição de que a imagem que cada indivíduo tem de si próprio e dos outros pode ser representada por dois pólos, um positivo e um negativo. Com base nestas dimensões, distinguem quatro estilos de vinculação: o seguro (com uma visão de si e dos outros positiva), o desinvestido (com uma visão de si próprio positiva e negativa dos outros), o preocupado (com uma visão de si negativa, mas positiva dos outros) e o amedrontado (com modelos negativos de si e dos outros).

$\mathrm{Na}$ investigação, existe uma grande evidência da vinculação segura como factor protector relativamente ao desenvolvimento de perturbações psicológicas e da vinculação insegura como um factor de risco (Allen, Moore, Kuperminc \& Bell, 1998; Cowan, Cohn, Cowan \& Pearson, 1996; Crowell, 2003; Kochanska, 2001; Rutter, 1999; Sroufe, Carlson, Levy \& Egeland, 1999; Thompson, 2001; Wartner, Grossmann, Fremmer-Bombik \& Suess, 1994; Weinfield, Sroufe, Egeland \& Carlson, 1999).

A qualidade dos laços afectivos estabelecidos com as figuras de vinculação, desde a infância, está associada a maior confiança em si próprio e nos outros, maior sociabilidade e competência e melhor controle emocional (Bowlby, 1973; Greenberg, 1999). A investigação tem mostrado que a qualidade do afecto dos adolescentes e jovens aos pais está relacionada positivamente com a satisfação dos indivíduos consigo próprios, maior auto-estima e melhor adaptação a vários contextos de vida (Allen, Moore, Kuperminc \& Bell, 1998; Lapsley, Rice \& Fitzgerald, 1990; Paterson, Pryor \& Field, 1995; Raja, McGee, \& Stanbon, 1991). A relação com os pais, neste período, parece funcionar como uma base segura através da qual os adolescentes podem explorar e dominar o seu meio, de forma autónoma (Allen \& Land, 1999; Armsden \& Greenberg, 1987), o que permitirá o 
desenvolvimento de um sentimento de autoconfiança e de competência pessoal e social (Allen, et al., 1998; Paterson et al., 1995).

Quando, pelo contrário, os laços afectivos construídos com as figuras de vinculação se caracterizam por inconsistência, rejeição, supercontrole e inibição da exploração conduzem a falta de confiança em si próprio e nos outros, a um sentimento de desvalorização pessoal e a baixa capacidade de controle emocional (Greenberg, 1999). Deste modo, os adolescentes sentem-se mais dependentes dos pais e mais ameaçados com a separação das figuras de vinculação (Marsh, McFarland, Allen, McElhaney \& Land, 2003).

Estudos realizados com jovens adultos verificaram que uma vinculação segura aos pais conduzia a uma visão de si próprio positiva e menos queixas sintomáticas perante acontecimentos de vida negativos (Armsden \& Greenberg, 1987). Os indivíduos com vinculação segura, comparativamente aos inseguros, tem maior capacidade para aceitar novas informações e adaptar-se a mudanças, apresentando, por isso, maior flexibilidade na sua forma de pensar e de agir (Lyddon \& Sherry, 2001).

No entanto, apesar dos estudos evidenciarem que laços emocionais dos jovens aos pais baseados em rejeição ou superproteção conduzem à disfuncionalidade a nível psicológico, são poucos os estudos que analisam especificamente a relação entre a vinculação e a ansiedade. Numa revisão da investigação neste domínio, realizada por Manassis (2001), são citados apenas três estudos (Manassis, Bradley, Goldberg, Hood \& Swinson, 1994; Cassidy, 1995; Warren, Huston, Egeland \& Carlson, 1997), cujas conclusões apontam, genericamente, para uma relação positiva entre a vinculação insegura e a ansiedade. O estudo de Warren et al. (1997), por exemplo, analisou, através de uma metodologia longitudinal, a associação entre estilos de vinculação na infância e na adolescência com ansiedade, concluindo que as crianças com estilo de vinculação ansioso/resistente (correspondente ao estilo preocupado no adulto) eram as mais propensas a apresentar perturbações de ansiedade na adolescência, comparativamente às crianças com estilos de vinculação segura ou evitante.

No mesmo sentido, estudos realizados no nosso país reforçam as conclusões das investigações anteriores. Canavarro (1999) realizou um estudo numa amostra clínica cujos resultados revelaram que os melhores preditores das perturbações de ansiedade na idade adulta são o facto de se ter sido rejeitado pelos pais na infância e na adolescência e de apresentar padrões de vinculação ansiosa na idade adulta.

Do mesmo modo, estudos realizados em amostras não clínicas revelam uma relação positiva entre insegurança e ansiedade. Por exemplo, um estudo realizado numa população universitária (Cunha \& Gouveia, 1999) analisou a influência das relações de vinculação relativamente à ansiedade 
social. Os dados deste estudo revelaram que os indivíduos com ansiedade social mais elevada apresentavam esquemas interpessoais, resultantes das regras estabelecidas com as figuras de vinculação, que os levavam a auto-representarem-se como frágeis e vulneráveis e a percepcionarem os outros como ameaçadores e pouco amistosos.

Os estilos de vinculação insegura apresentam, geralmente, problemas de relacionamento interpessoal, como uma preocupação excessiva com as relações, medo da intimidade, evitamento social e desinvestimento nos outros (Horowitz, Rosenberg \& Bartholomew, 1993). Estes indivíduos tem uma imagem de si próprios negativa, baixa auto-estima e relações interpessoais menos frequentes e menos satisfatórias, o que contribui para se sentirem mais ansiosos e incapazes em situações sociais, podendo ser mais susceptíveis de desenvolver medos sociais (Salvador, 1997; Sroufe, 1990).

Pela análise das várias investigações com base no modelo da vinculação, depreende-se a importância das relações precoces com pessoas significativas para o desenvolvimento de si próprio e da relação com os outros e com mundo. Relações construídas com base em confiança e segurança desde a infância são pilares para as etapas desenvolvimentais posteriores e parecem funcionar como factor protector para problemas emocionais como a ansiedade, sobretudo nos períodos de maior transição, como é o caso da juventude.

Assim, considerando o anteriormente exposto, este estudo pretende ter um carácter exploratório quanto à possível relação entre vinculação ao pai e à mãe e a ansiedades em jovens adultos. Neste sentido, formulámos as seguintes hipóteses: (1) Espera-se correlações significativas entre as dimensões da vinculação - qualidade de laço emocional, inibição da exploração e individualidade e ansiedade de separação e dependência - com os medos e características obsessivo-compulsivas (Allen et al., 1998; Greenberg, 1999; Marsh et al., 2003; Paterson et al., 1995); (2) Espera-se que o padrão de vinculação segura aos pais esteja negativamente relacionado com a ansiedade (Allen \& Land, 1999; Allen et al. 1998; Greenberg, 1999; Lapsley et al., 1990; Landy, 2002; Lyddon \& Sherry, 2001); (3) Espera-se que os padrões de vinculação insegura, particularmente o estilo de vinculação preocupado, aos pais esteja positivamente relacionado com a ansiedade (Canavarro, 1999; Cassidy \& Berlin, 1994; Cassidy, 1995; Cunha \& Gouveia, 1999; Manassis et al., 1994; Marsh et al., 2003; Warren et al., 1997).

Seguidamente, faz-se referência à metodologia utilizada neste trabalho, segue-se a análise e discussão dos resultados e, por último, reflecte-se acerca das implicações dos dados da investigação para a intervenção psicológica junto dos jovens. 


\section{Metodologia}

\section{Constituição da amostra}

A amostra é constituída por 511 alunos universitários, de diferentes cursos de estabelecimentos de ensino superior da Universidade do Porto, sendo 363 raparigas $(71 \%)$ e 148 rapazes $(29 \%)$, com idades compreendidas entre os 17 e os 25 anos (média $=20,89 \mathrm{DP}=1,88$ ). A construção da amostra foi realizada de forma aleatória, conforme a disponibilidade dos professores.

\section{Instrumentos}

Este estudo utilizou os seguintes instrumentos de auto-relato:

- Questionário demográfico. Construído para o estudo, para recolha de informação acerca de algumas características dos estudantes.

- Fear Survey Scale (FSS; Wolpe \& Lazarus, 1966). Esta escala é composta por 75 itens que teoricamente correspondem aos estímulos fóbicos mais temidos. O sujeito pode situar a sua auto-avaliação numa escala de Likert de 1-5 em que o valor 1 correspondente a não ter medo nenhum, até ao valor 5 , indicador de ter bastante medo. Foi realizada uma análise factorial, pelo método Principal Component Analysis com rotação Varimax (método utilizado em todas as escalas deste estudo), resultando 10 factores que explicam 64,46 \% da variância total: factor 1 (rejeição social) explica $24,40 \%$ do total de variância, $\operatorname{com} \alpha=0,89$; factor 2 (animais) explica 8,40 do total de variância, com $\alpha=0,84$; factor 3 (sangue/ferimentos) explica 5,50 do total de variância, com $\alpha=0,80$; factor 4 (violência) explica 4,86\% do total de variância, com $\alpha=0,77$; factor 5 (viagens) explica 4,68\% do total de variância, com $\alpha=0,69$; factor 6 (mau humor) explica 3,90\% do total de variância, com $\alpha=0,79$; factor 7 (alturas) explica 3,42\% do total de variância, $\operatorname{com} \alpha=0,93$; factor 8 (pessoas desagradáveis) explica 3,23\% do total de variância, $\operatorname{com} \alpha=0,92$; factor 9 (mau tempo) explica 3,22\% do total de variância, com $\alpha=0,63$; e o factor 10 (exposição social) explica $2,96 \%$ do total de variância, $\operatorname{com} \alpha=0,63$.

- Fear Questionnaire (FQ; Marks \& Mathews, 1979) É um questionário de auto-avaliação da ansiedade fóbica constituído por três partes: a primeira parte é relativa à avaliação da fobia mais importante do sujeito; a segunda parte é constituída por 3 subescalas: agorafobia, fobia social e fobia a sangue e ferimentos; a terceira parte contém questões para avaliar o grau de ansiedade e depressão (Emmelkamp, Bowmann, \& Scholing, 1992). Os sujeitos avaliam o quanto evitam um determinado item fóbico 
(correspondente ao estímulo que teme) ou grau de ansiedade, numa escala de Likert de 0-8.

Da análise factorial efectuada na nossa amostra, resultaram 3 factores: o factor 1 (agorafobia) explica $26,40 \%$ do total de variância, com $\alpha=0,74$; o segundo factor (fobia a sangue/ferimentos) explica $12,91 \% \mathrm{com}$ $\alpha=0,62$; e o terceiro factor (fobia social) explica $9,00 \%$, com $\alpha=0,65$.

Uma vez que estes dois questionários (FSS e FQ) permitiam avaliar medos, realizou-se uma correlação entre os seus factores, no sentido de avaliar de que forma se relacionavam. Obtivemos valores baixos de correlação entre os factores dos dois questionários, o que parece indicar que avaliam medos diferentes, no entanto, salientam-se duas excepções: (1) correlações moderadas $(r=0,422$ e $r=0,484)$ entre o factor 3 (fobia social) do FQ e o factor 1 (medo de rejeição social) e o factor 10 (medo de exposição social) do FSS, que parecem avaliar o mesmo tipo de medo. Sendo assim, e para simplificar a nossa análise, utilizaremos apenas o factor fobia social do FQ, que parece avaliar quer a rejeição quer a exposição social; (2) - do mesmo modo, assistiu-se a uma correlação elevada $(r=0,711)$ entre o factor 1 (sangue/ferimentos) do FQ e o factor 3 do FSS (sangue/ferimentos), que avaliam o mesmo medo. Assim, optou-se por analisar o factor 1 do FQ para a avaliação do medo a sangue e a ferimentos, uma vez que, na correlação anterior, também optámos pelo factor deste questionário.

- Padua Inventory (PI; Sanavio, 1988). Este instrumento de auto-relato consiste num inventário de 60 itens que descrevem os comportamentos obsessivos e compulsivos mais comuns. Cada item é avaliado numa escala de Likert de 0-4, em que o zero indica que não existe qualquer perturbação e 4 indica um grau elevado de perturbação. Da análise factorial realizada, emergiram 4 factores: factor 1 (pensamentos obsessivos) que explica $27,51 \%$ do total de variância, com $\alpha=0,93$; factor 2 (impulsos obsessivos) que explica 7,83\% do total de variância, com $\alpha=0,89$; factor 3 (compulsões de verificação) com 5,67\% e um $\alpha=0,88$; e o factor 4 (compulsões de limpeza) que explica $4,32 \%$ do total de variância, com $\alpha=0,84$.

- Questionário da Vinculação ao pai e à mãe (QVPM; Matos, Almeida \& Costa, 1997). A versão utilizada é constituída por uma escala com 31 itens, organizados separadamente para o pai e para a mãe, em dimensões baseadas numa abordagem dimensional da vinculação adulta. $\mathrm{O}$ instrumento pretende medir as percepções dos adolescentes e jovens adultos acerca das relações de vinculação que mantêm com a mãe e com o pai. Os sujeitos respondem em escalas de Likert de 6 níveis, de acordo com o que pensam e sentem acerca da relação com cada um dos pais (1 - "concordo totalmente" e 6 - "discordo totalmente"). Este questionário foi validado em 
várias amostras portuguesas (Matos, Almeida, \& Costa, 1997, 1999; Matos, Barbosa \& Costa, 2001; Barbosa, 2001; Matos, 2002). Na nossa amostra, a análise factorial evidenciou 3 factores, previstos na estrutura dos instrumento: qualidade de laço emocional (QLE - factor 1) que explica $16,52 \%$ do total da variância, com $\alpha=0,90$; inibição da exploração $e$ individualidade (IEI - factor 2) que explica $14,17 \%$ do total de variância, $\operatorname{com} \alpha=0,81$; e um terceiro factor, designado por ansiedade de separação e dependência (ASD), que explica $13,92 \%$ do total de variância, com $\alpha=0,79$.

\section{Apresentação dos resultados e discussão}

\section{Relação entre dimensões da vinculação e ansiedade}

Para avaliar a relação existente entre as dimensões da vinculação e os diferentes tipos de medo e características obsessivo-compulsivas, foram realizadas correlações de Pearson, cujos resultados passamos a descrever.

Quadro 1: Correlações de Pearson entre as dimensões da vinculação à mãe e os medos e características obsessivo-compulsivas

\begin{tabular}{ll|c|c}
\hline \multirow{2}{*}{$\begin{array}{l}\text { Medos e características } \\
\text { obsessivo-compulsivas }\end{array}$} & $\begin{array}{c}\text { Qualidade de } \\
\text { laço emocional }\end{array}$ & $\begin{array}{c}\text { Inibição da exploração } \\
\text { e individualidade }\end{array}$ & $\begin{array}{c}\text { Ansiedade de } \\
\text { separação }\end{array}$ \\
\hline Medos & & & \\
Agorafobia & $0,090^{*}$ & 0,011 & $0,314^{* *}$ \\
Fobia social & 0,021 & $0,110^{*}$ & $0,250^{* *}$ \\
Medo de sangue/ferimentos & 0,037 & $-0,011$ & $0,172^{* *}$ \\
Medo de animais & $0,149^{* *}$ & $-0,030$ & $0,275^{* *}$ \\
Medo de violência & $0,113^{*}$ & 0,022 & $0,216^{* *}$ \\
Medo de viagens & $0,200^{* *}$ & $0,093^{*}$ & $0,600^{* *}$ \\
Medo de alturas & 0,030 & 0,056 & $0,159^{* *}$ \\
Medo de pessoas desagradáveis & 0,011 & $-0,025$ & $0,206^{* *}$ \\
Medo de mau tempo & $0,109^{*}$ & $-0,014$ & $0,217^{* *}$ \\
Medo de mau humor & $-0,050$ & $0,090^{*}$ & $0,091^{*}$ \\
Características obsessivo-compulsivas & & & \\
Pensamentos obsessivos & $-0,100^{*}$ & $0,289^{* *}$ & $0,241^{* *}$ \\
Impulsos obsessivos & $-0,142^{* *}$ & $0,172^{* *}$ & 0,085 \\
Compulsões de verificação & $-0,050$ & $0,144^{* *}$ & $0,179^{* *}$ \\
Compulsões de limpeza & 0,038 & $0,091^{*}$ & $0,170^{* *}$ \\
\hline
\end{tabular}

$* * \mathrm{p}<0,001, * \mathrm{p}<0,05$ 
A análise do quadro 1 permite constatar a inexistência de correlações significativas entre a qualidade de laço emocional e os medos, com as seguintes excepções: (1) o medo de animais, medo de violência e medo de mau tempo, que apresentam correlações significativas baixas; (2) os pensamentos obsessivos, que apresentam uma correlação negativa e baixa, ou seja, quanto maior for a qualidade de laço emocional menor os pensamentos obsessivos. No que respeita às correlações entre a inibição da exploração e individualidade e a ansiedade, constata-se, também, a inexistência de correlações significativas relativamente aos medos, com excepção da fobia social e medo de mau humor, que apresentam correlações baixas. Assiste-se, porém, a correlações significativas baixas a moderadamente baixas desta dimensão com as características obsessivo-compulsivas, exceptuando as compulsões de limpeza. Por último, podemos ainda observar no quadro a existência de correlações significativas positivas baixas e moderadas entre a ansiedade de separação e dependência e a ansiedade, exceptuando-se apenas o medo de mau humor e os impulsos obsessivos.

A estrutura das correlações de Pearson entre as dimensões da vinculação ao pai e a ansiedade (quadro 2) são, na generalidade, semelhantes às descritas anteriormente. Assim, pela leitura do quadro 2 constata-se que a qualidade de laço emocional ao pai apresenta apenas uma correlação significativa baixa com o medo a animais e correlações negativas baixas com os pensamentos e impulsos obsessivos. Podemos ainda observar que a inibição da exploração e individualidade apresenta correlações baixas na fobia social e medo de mau humor e correlações baixas ou moderadamente baixas com as características obsessivas. Quanto à ansiedade de separação e dependência, constata-se a existência de correlações significativas baixas ou moderadas com os medos, exceptuando-se apenas o medo de mau humor, e assiste-se ainda a correlações significativas baixas com as características obsessivo-compulsivas, exceptuando os impulsos obsessivos.

Em conjunto, os valores de correlação entre a qualidade de laço emocional ao pai e à mãe com os medos e com as características de obsessividade são baixos, não confirmando a nossa hipótese, ainda que a literatura enfatize a importância da qualidade dos laços afectivos entre pais e filhos, desde a infância e ao longo do desenvolvimento, para a aquisição de confiança em si próprios e nos outros (Bowlby, 1973; Erikson, 1968; Greenberg, 1999), contribuindo para a construção de uma visão do mundo como menos perigoso e para que a separação das figuras de vinculação seja vivida de forma menos ameaçadora e ansiosa. Talvez estes resultados possam indicar que esta dimensão da vinculação não é a mais relevante para explicar o desenvolvimento da ansiedade. 
Quadro 2: Correlações de Pearson entre as dimensões da vinculação ao pai e os medos e características obsessivo-compulsivas

\begin{tabular}{ll|c|c}
\hline \multirow{2}{*}{$\begin{array}{l}\text { Medos e características } \\
\text { obsessivo-compulsivas }\end{array}$} & \multicolumn{2}{c}{ Dimensões da vinculação } \\
\cline { 2 - 4 } & $\begin{array}{c}\text { Qualidade de laço } \\
\text { emocional }\end{array}$ & $\begin{array}{c}\text { Inibição da explo- } \\
\text { ração e individuali- } \\
\text { dade }\end{array}$ & $\begin{array}{c}\text { Ansiedade de } \\
\text { separação }\end{array}$ \\
\hline Medos & 0,038 & 0,041 & $0,295^{* *}$ \\
Agorafobia & $-0,016$ & $0,151^{*}$ & $0,212^{* *}$ \\
Fobia social & $-0,008$ & 0,003 & $0,139^{* *}$ \\
Medo de sangue/ferimentos & $0,088^{*}$ & 0,000 & $0,275^{* *}$ \\
Medo de animais & 0,021 & 0,049 & $0,165^{* *}$ \\
Medo de violência & 0,060 & $0,127^{* *}$ & $0,486^{* *}$ \\
Medo de viagens & 0,020 & 0,044 & $0,156^{* *}$ \\
Medo de alturas & 0,016 & 0,003 & $0,191^{* *}$ \\
Medo de pessoas desagradáveis & 0,039 & $0,093^{*}$ & $0,195^{* *}$ \\
Medo de mau tempo & $-0,012$ & $0,136^{* *}$ & 0,081 \\
Medo de mau humor & & & $0,199^{* *}$ \\
Características obsessivo-compulsivas & $-0,111^{*}$ & $0,282^{* *}$ & 0,081 \\
Pensamentos obsessivos & $-0,111^{*}$ & $0,179^{* *}$ & $0,172^{* *}$ \\
Impulsos obsessivos & $-0,035$ & $0,173^{* *}$ & $0,157^{* *}$ \\
Compulsões de verificação & 0,003 & $0,113^{*}$ & \\
Compulsões de limpeza & & & \\
\hline
\end{tabular}

** $\mathrm{p}<0,001, * \mathrm{p}<0,05$

Do mesmo modo, verifica-se que as da inibição da exploração e individualidade à mãe e ao pai com os medos e com as características obsessivas e compulsivas são genericamente baixas. A inibição da exploração conduz a uma dependência face aos outros, principalmente das figuras de vinculação e, ao mesmo tempo, no medo de arriscar em novas relações (fobia social, medo de mau humor) e de se afastar dos locais conhecidos (medo de viajar). Quando os pais não facilitam a aquisição da autonomia, por exemplo, exercendo um poder supercontrolador e inibidor da exploração, surge uma propensão para a dúvida e para a vergonha, relacionadas com a incapacidade de não se dominar a si próprio, que pode construir-se como um medo de perder o controle e traduzir-se em preocupações obsessivas e compulsivas (Costa, $\mathrm{s} / \mathrm{d}$ ). Pelo contrário, quando os pais servem de base segura, favorecem a autonomia do jovem e ao mesmo tempo promovem o seu desenvolvimento pessoal e interpessoal de modo ajustado (Allen et al., 1998; Paterson et al., 1995). Dada a relevância da aquisição da autonomia para o jovem adulto, seriam de esperar correlações significativas mais elevadas entre a inibição das exploração e individualidade e ansiedade. No entanto, estes resultados poderão indicar que esta dimensão da vinculação, tal como a qualidade de laço emocional, sendo de grande impor- 
tância para o desenvolvimento da visão de si próprio e da relação com o mundo, possa não se relacionar de modo directo a emoção de ansiedade

Assiste-se ainda, a correlações significativas positivas da ansiedade de separação à mãe e ao pai com a ansiedade. Assim, de modo geral, o sentido destas correlações confirma as expectativas, isto é, valores elevados na dimensão ansiedade de separação e dependência estão associados a maior ansiedade. Esta ansiedade traduz o medo da perda ou da ausência da figura de vinculação, característica da vinculação ansiosa, o que leva o sujeito a tornar-se dependente desta figura, no sentido de a monitorizar e de a ter acessível (Kobak, 1999). Segundo a literatura, esta ansiedade de separação e dependência é particularmente evidente no estilo de vinculação preocupado, em que o modelo de si próprio é frágil e negativo e o modelo dos outros é positivo. Os indivíduos com este padrão de vinculação sentem que precisam dos outros para sobreviver (Lyddon \& Sherry, 2001). Esta dimensão da vinculação aparece igualmente associada à fobia escolar nas crianças e adolescentes e à agorafobia no jovem adulto e adulto (Bowlby, 1973; Routh \& Bernholtz, 1991). Assim, estes resultados parecem revelar que a ansiedade de separação e dependência é a dimensão da vinculação aos pais que mais se relaciona com a ansiedade.

Em suma, o que os nossos resultados permitem salientar é que, nesta fase de transição e, portanto, de maior insegurança e vulnerabilidade, ainda que a qualidade de laço emocional e a inibição da exploração e individualidade apresentem algumas correlações com os medos e as características obsessivo-compulsivas, é a ansiedade de separação e dependência em relação aos pais que apresenta correlações mais significativas com a ansiedade.

\section{Estilos de vinculação e ansiedade}

Para analisar a relação entre os quatro protótipos de vinculação ao pai e à mãe (seguro, preocupado, amedrontado e desinvestido) e os medos e características obsessivas e compulsivas como variáveis dependentes, foram realizadas MANOVAS (com o sexo como covariável, medida em que a variável género apresenta efeitos significativos com a ansiedade).

Inicialmente, foi realizada uma análise de clusters $(\mathrm{K}$ - means e simple euclidian distance) com o objectivo de avaliar a existência de configurações específicas na organização das dimensões da vinculação. Pretende-se verificar em que medida os padrões de resultados que definem os diferentes clusters de indivíduos são consistentes com o modelo bidimensional de Bartholomew (1990; Bartholomew \& Horowitz, 1991). Esperava-se que os valores das várias dimensões (qualidade de laço emocional, inibição da exploração e individualidade e ansiedade de separação e dependência) se 
organizassem de modo a evidenciar os quatro protótipos de vinculação: seguro, preocupado, amedrontado e desinvestido. Os sujeitos foram, então, distribuídos por 4 clusters, correspondendo cada um a um protótipo de vinculação (quadro 3 e quadro 4 ).

Como se verifica nos quadros 3 e 4, os resultados parecem evidenciar a possibilidade dos clusters serem interpretados à luz do modelo de vinculação de Bartholomew (1990, Bartholomew \& Horowitz, 1991). Assim, no cluster 1 (versão mãe e pai) encontram-se os sujeitos com elevados níveis de qualidade de laço emocional, menor grau de inibição da exploração e individualidade e com graus moderados de ansiedade de separação e dependência. Este cluster parece evidenciar o protótipo de vinculação segura.

No cluster 2 observa-se que os valores de ansiedade de separação e dependência são notoriamente os mais elevados relativamente aos outros grupos, verificando-se uma boa qualidade de laço emocional. Estes sujeitos, apesar de confiarem nas figuras parentais, parecem depender delas. Assim, estes valores parecem definir o protótipo de vinculação preocupado.

Quadro 3: Análise de clusters do QVPM - versão mãe

\begin{tabular}{l|c|c|c|c}
\hline \multirow{2}{*}{ Dimensões } & \multicolumn{4}{|c}{ Clusters } \\
& Cluster 1 & Cluster 2 & Cluster 3 & Cluster 4 \\
\cline { 2 - 5 } & Seguro & $\begin{array}{c}\text { Preocupado } \\
(\mathrm{n}=155)\end{array}$ & $\begin{array}{c}\text { Desinvestido } \\
(\mathrm{n}=25)\end{array}$ & $\begin{array}{c}\text { Amedrontado } \\
(\mathrm{n}=144)\end{array}$ \\
\hline \multirow{2}{*}{ QLE } & 54,53 & 56,34 & 37,28 & 51,23 \\
IEI & 16,23 & 20,31 & 34,52 & 29,14 \\
ASD & 23,38 & 35,15 & 19,08 & 25,54 \\
\hline
\end{tabular}

Quadro 4: Análise de clusters do QVPM - versão pai

\begin{tabular}{l|c|c|c|c}
\hline \multirow{2}{*}{ Dimensões } & \multicolumn{4}{|c}{ Clusters } \\
& Cluster 1 & Cluster 2 & Cluster 3 & Cluster 4 \\
\cline { 2 - 5 } & Seguro & Preocupado & Desinvestido & Amedrontado \\
& $(\mathrm{n}=187)$ & $(\mathrm{n}=155)$ & $(\mathrm{n}=25)$ & $144)$ \\
\hline \multirow{2}{*}{ QLE } & 53,79 & 55,13 & 28,70 & 47,67 \\
IEI & 15,75 & 20,73 & 32,68 & 28,58 \\
ASD & 22,94 & 33,56 & 16,33 & 24,08 \\
\hline
\end{tabular}


Em contraste com este, o terceiro cluster reúne os indivíduos que apresentam valores reduzidos de ansiedade de separação e dependência. $\mathrm{O}$ facto de estes sujeitos se caracterizarem por uma ansiedade de separação tão baixa comparativamente aos outros grupos parece apontar para o grupo dos indivíduos com vinculação desinvestida.

Por último, o quarto cluster reúne os sujeitos com valores de inibição de exploração e individualidade mais elevados do que os clusters 1 e 2 (vinculação à mãe e ao pai). Apresentam ainda valores de ansiedade de separação e dependência mais elevados do que o grupo dos desinvestidos e seguros. Estes resultados parecem evidenciar o protótipo dos amedrontados, que se caracterizam por uma reduzida autonomia e uma ansiedade de separação elevada, embora menor que os sujeitos do grupo preocupado.

Posteriormente, para analisar a relação entre os estilos de vinculação e variáveis da ansiedade, realizaram-se análises de variância e teste "post hoc" de Scheffé. Os resultados destas análises indicam um efeito significativo da vinculação ao pai e à mãe relativamente às variáveis dependentes que passamos a descrever de seguida.

Vinculação à mãe e ansiedade

Constata-se que na vinculação à mãe existem diferenças de médias estatisticamente significativas no que respeita aos medos e características obsessivas e compulsivas $[\mathrm{F}(42,1485)=4,787, \mathrm{p}<0,001]$. No quadro 5, podemos observar as diferenças de diferenças de médias estatisticamente significativas.

\section{Vinculação ao pai e ansiedade}

Verifica-se que existem diferenças de médias estatisticamente significativas da vinculação ao pai relativamente às variáveis dependentes em estudo (medos e características obsessivo-compulsivas), $[\mathrm{F}(42,1485)=$ $3,748, \mathrm{p}<0,001]$. No quadro seguinte evidenciam-se as diferenças de médias estatisticamente significativas.

Constatamos que a vinculação à mãe e ao pai apresenta diferenças de médias estatisticamente significativas, relativamente aos vários tipos de medo e características obsessivo-compulsivas. Tal como era esperado, os indivíduos com estilo de vinculação insegura, particularmente o estilo de vinculação preocupado, são os que apresentam médias mais elevadas nos vários tipos de ansiedade. 
Quadro 5: Análise de variância do estilo de vinculação à mãe relativamente aos medos e às características obsessivo-compulsivas

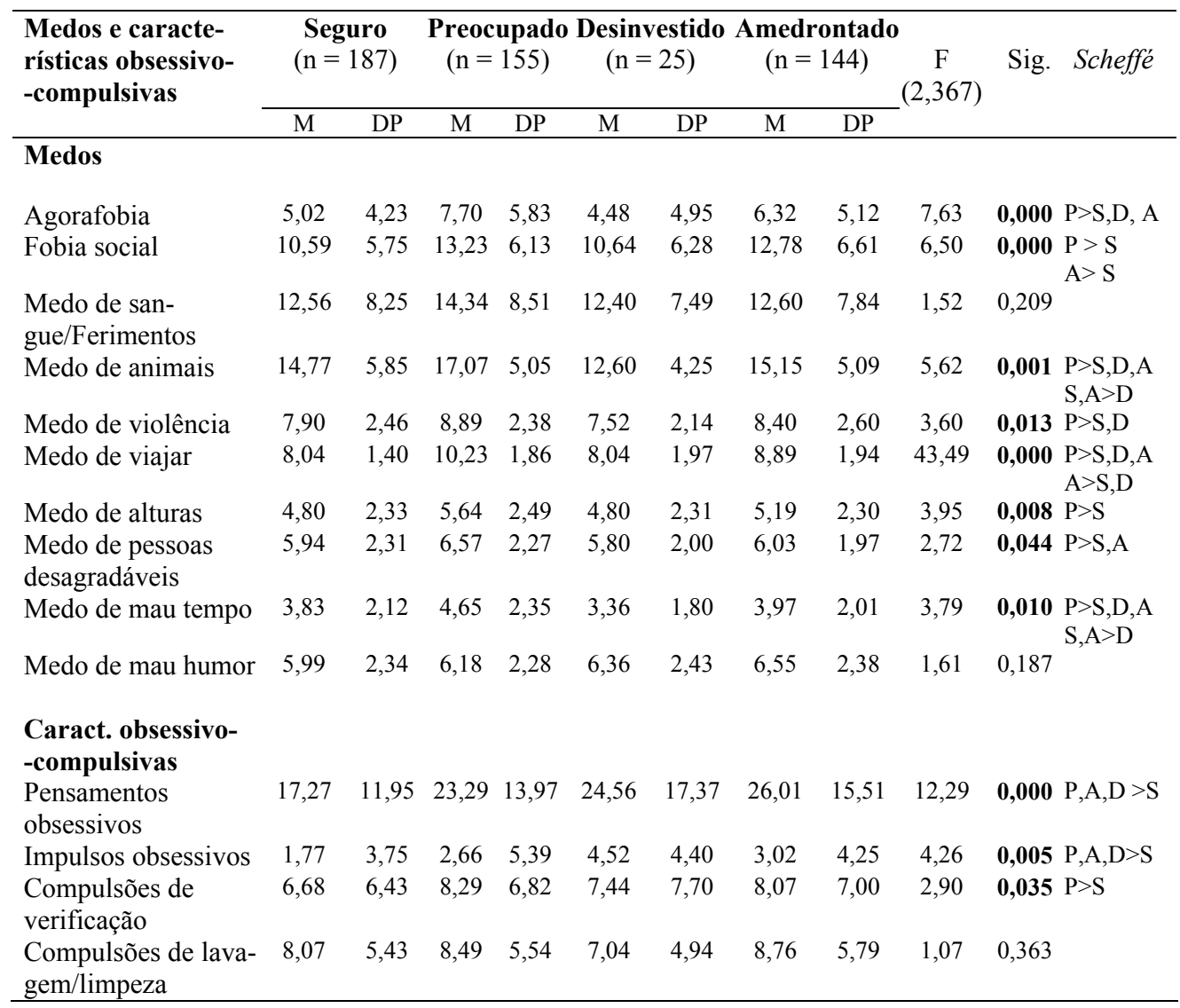

Nota: S (seguro), P (preocupado), D (desinvestido), A (amedrontado)

Pela análise dos quadros 5 e 6, podemos constatar que os indivíduos do estilo preocupado assumem médias significativamente mais elevadas nos medos, comparativamente aos restantes grupos. Estes dados confirmam os resultados de outras investigações que têm revelado que o estilo de vinculação preocupado (resistente/ambivalente) é o mais propenso para desenvolver perturbações de ansiedade (Landy, 2002; Cassidy, 1995; Cassidy \& Berlin, 1994; Warren et al., 1997). Os sujeitos com este padrão de vinculação desenvolvem uma excessiva preocupação relativamente à figura de vinculação e, simultaneamente uma grande necessidade de a monitorizar. 
Quadro 6: Análise de variância do estilo de vinculação ao pai relativamente aos medos e às características obsessivo-compulsivas

\begin{tabular}{|c|c|c|c|c|c|c|c|c|c|c|}
\hline \multirow[t]{2}{*}{$\begin{array}{l}\text { Medos e caracte- } \\
\text { rísticas obsessivo- } \\
\text {-compulsivas }\end{array}$} & \multicolumn{2}{|c|}{$\begin{array}{l}\text { Seguro } \\
(\mathrm{n}=187)\end{array}$} & \multicolumn{2}{|c|}{$\begin{array}{l}\text { Preocupado } \\
(\mathrm{n}=155)\end{array}$} & \multicolumn{4}{|c|}{$\begin{array}{l}\text { Desinvestido Amedrontado } \\
\quad(\mathrm{n}=25) \quad(\mathrm{n}=144)\end{array}$} & \multirow[t]{2}{*}{$\begin{array}{c}F \\
(2,367)\end{array}$} & \multirow[t]{2}{*}{ Scheffé } \\
\hline & $\mathrm{M}$ & $\mathrm{DP}$ & $\mathrm{M}$ & DP & $\mathrm{M}$ & $\mathrm{DP}$ & $\mathrm{M}$ & $\mathrm{DP}$ & & \\
\hline \multicolumn{11}{|l|}{ Medos } \\
\hline Agorafobia & 4,93 & 4,33 & 7,73 & 5,67 & 5,30 & 5,88 & 6,25 & 4,92 & 8,29 & $\mathbf{0 , 0 0 0} \mathrm{P}>\mathrm{S}, \mathrm{D}, \mathrm{A}$ \\
\hline Fobia social & 10,6 & 6,08 & 13,67 & 6,15 & 11,88 & 5,65 & 12,03 & 6,29 & 7,02 & $\mathbf{0 , 0 0 0} \mathrm{P}>\mathrm{S}, \mathrm{A}$ \\
\hline $\begin{array}{l}\text { Medo de san- } \\
\text { gue/feriemntos }\end{array}$ & 12,66 & 8,13 & 13,81 & 8,39 & 12,94 & 9,25 & 12,91 & 7,83 & 0,53 & 0,663 \\
\hline Medo a animais & 14,52 & 5,58 & 16,92 & 5,30 & 13,85 & 5,03 & 15,46 & 5,20 & 6,40 & $\begin{aligned} \mathbf{0 , 0 0 0} & \begin{array}{l}\mathrm{P}>\mathrm{S}, \mathrm{D}, \mathrm{A} \\
\mathrm{A}\end{array}\end{aligned}$ \\
\hline Medo de violência & 7,92 & 2,44 & 8,71 & 2,48 & 8,34 & 2,50 & 8,41 & 2,53 & 2,05 & 0,106 \\
\hline Medo de viajar & 8,20 & 1,54 & 9,98 & 1,79 & 8,36 & 2,57 & 8,88 & 1,97 & 26,99 & $\mathbf{0 , 0 0 0} \mathrm{P}>\mathrm{S}, \mathrm{D}, \mathrm{A}$ \\
\hline Medo de alturas & 4,77 & 2,38 & 5,52 & 2,40 & 4,73 & 2,25 & 5,40 & 2,36 & 3,75 & $\begin{array}{rl}0,011 & \mathrm{P}>\mathrm{S} \\
\mathrm{A} & >\mathrm{S}\end{array}$ \\
\hline $\begin{array}{l}\text { Medo de pessoas } \\
\text { desagradáveis }\end{array}$ & 5,95 & 2,31 & 6,45 & 2,24 & 5,89 & 2,37 & 6,14 & 1,95 & 1,60 & 0,193 \\
\hline $\begin{array}{l}\text { Medo de mau } \\
\text { tempo }\end{array}$ & 3,65 & 1,98 & 4,63 & 2,32 & 4,03 & 2,20 & 4,10 & 2,15 & 4,83 & $0,003 \quad P>S$ \\
\hline $\begin{array}{l}\text { Medo de mau } \\
\text { humor }\end{array}$ & 5,91 & 2,26 & 6,28 & 2,33 & 5,94 & 2,11 & 6,63 & 2,46 & 2,71 & $0,044 \mathrm{~A}>\mathrm{S}$ \\
\hline \multicolumn{11}{|l|}{$\begin{array}{l}\text { Caract. obsessivo- } \\
\text {-compulsivas }\end{array}$} \\
\hline $\begin{array}{l}\text { Pensamentos obses- } \\
\text { sivos }\end{array}$ & 16,25 & 10,81 & 24,29 & 14,50 & 23,33 & 14,06 & 26,38 & 16,02 & 17,64 & $\mathbf{0 , 0 0 0} \mathrm{P}, \mathrm{D}, \mathrm{A}>\mathrm{S}$ \\
\hline Impulsos obsessivos & 1,54 & 3,22 & 2,80 & 5,36 & 2,88 & 3,09 & 3.44 & 4.99 & 5,52 & $\mathbf{0 , 0 0 1} \mathrm{P}, \mathrm{A}>\mathrm{S}$ \\
\hline $\begin{array}{l}\text { Compulsões de } \\
\text { verificação }\end{array}$ & 5,94 & 6,09 & 8,72 & 6,81 & 7,17 & 6,29 & 8.63 & 7.38 & 7,50 & $\mathbf{0 , 0 0 0} \mathrm{P}, \mathrm{A}>\mathrm{S}$ \\
\hline $\begin{array}{l}\text { Compulsões de la- } \\
\text { vagem/limpeza }\end{array}$ & 7,49 & 5,30 & 8,96 & 5,71 & 8,03 & 5,30 & 8.85 & 5.62 & 2,95 & $\mathbf{0 , 0 3 2} \mathrm{P}, \mathrm{A}>\mathrm{S}$ \\
\hline
\end{tabular}

Nota: S (seguro), P (preocupado), D (desinvestido), A (amedrontado)

Estas atitudes desenvolvem-se como resposta à inconsistência da figura de vinculação, que umas vezes dá suporte outras vezes está ausente (Landy, 2002). Estas preocupações conduzem a uma menor exploração do meio (Cassidy \& Berlin, 1994), a uma maior inibição e, consequentemente, menor autonomia, tornando estes indivíduos mais propensos à ansiedade de separação (agorafobia, medo de viajar, medo de alturas) e mais vulneráveis e medrosos perante situações desconhecidas ou difíceis de controlar (fobia social, medo de violência, medo de pessoas desagradáveis, medo de animais, medo de mau tempo). É ainda de salientar, como observamos na constituição dos clusters, que o estilo de vinculação preocupado é o que evidencia valores mais elevados na ansiedade de separação e dependência 
e é esta dimensão a que mais se correlaciona com a ansiedade, o que pode explicar as médias mais elevadas dos "preocupados" comparativamente aos outros grupos em vários tipos de ansiedade.

A análise dos resultados permite-nos ainda constatar que os indivíduos do grupo caracterizado por um estilo de vinculação amedrontado apresentam diferenças estatisticamente significativas, comparativamente aos indivíduos seguros na fobia social, e medo de viajar na vinculação à mãe, e o medo das alturas e medo de mau humor na vinculação ao pai. Estes dados permitem evidenciar o medo e ansiedade vivenciados por estes indivíduos (com modelos de si próprios e dos outros negativos), no confronto com situações sociais e interpessoais (fobia social e medo de mau humor) e no afastamento das suas figuras protectoras (medo de alturas e medo de viajar). Curiosamente, são estes indivíduos, a seguir aos que apresentam um estilo de vinculação preocupado, os que revelam valores mais elevados de ansiedade de separação e dependência, o que poderá explicar que este grupo também apresente níveis elevados de ansiedade.

Quanto às características obsessivo-compulsivas, verifica-se uma diferença de médias significativa dos grupos dos inseguros comparativamente aos seguros. Contrariamente ao que se esperava, o grupo dos indivíduos do estilo de vinculação preocupado não se evidencia dos outros grupos de vinculação insegura. De facto, alguns autores (Guidano, 1991; Guidano \& Liotti, 1983) defendem que a organização obsessivo-compulsiva parece estar associada a uma vinculação ambivalente em que as atitudes de rejeição são ocultadas por uma fachada de preocupação. Se, por um lado, os pais se dedicam à criança demonstrando o seu afecto, por outro lado, não exibem manifestações verbais e não verbais de amor. Assim, o indivíduo internaliza duas mensagens contraditórias, "ele ama-me" e "ele é indiferente comigo", o que o leva a estruturar uma atitude rígida para consigo próprio (Guidano \& Liotti, 1983). No entanto, outros autores (Joffe, Swison \& Regan, 1988; Steketee, 1993) salientam a importância do tipo de personalidade para este tipo de ansiedade e referenciam as personalidades evitante e dependente como as mais frequentes nos indivíduos obsessivos. Talvez o facto de os indivíduos com estilos desinvestido e amedrontado serem caracterizados pelo evitamento do relacionamento interpessoal (modelo negativo dos outros), e os indivíduos do estilo preocupado tenderem a depender dos outros (modelo positivo), possa explicar a inexistência de diferenças significativas entre estes grupos nas características obsessivas, comparativamente ao grupo dos seguros.

Em suma, os nossos resultados confirmam genericamente as nossas hipóteses e reforçam os resultados de outras investigações que evidenciam a vinculação insegura, mais especificamente o padrão preocupado de vinculação, como um factor de vulnerabilidade para a ansiedade. Consideran- 
do a importância destes resultados para a prevenção e tratamento da ansiedade, delineamos, de seguida, alguns aspectos que consideramos relevantes para a intervenção psicológica.

\section{Implicações para a intervenção psicológica}

Esta investigação tem implicações para a intervenção e tratamento das perturbações da ansiedade. Como ficou evidente, são os jovens com estilo de vinculação insegura aos pais, mais concretamente os classificados com um estilo preocupado e amedrontado, que apresentam maiores riscos para vir a desenvolver uma estrutura ansiosa. Assim, a intervenção psicológica deve orientar-se, de modo genérico, no sentido de promover a exploração e reconstrução dos modelos de si próprio e dos outros, de modo a que o jovem se sinta mais autoconfiante e seguro para explorar e agir autonomamente e construir uma visão de si próprio positiva e uma visão dos outros e do mundo menos ameaçadora e perigosa.

Neste estudo, verificámos que a dimensão da vinculação que mais se correlacionava com a ansiedade era a ansiedade de separação e dependência, constatámos também que são os indivíduos com um estilo de vinculação preocupado, seguidos dos indivíduos com estilo de vinculação amedrontado, os que apresentavam valores mais elevados nesta dimensão. Por outro lado, a análise da literatura revelou-nos que a ansiedade de separação se constrói em relações que superprotegem ou rejeitam o indivíduo, tornado-o vulnerável, frágil e dependente das figuras protectoras. Com base nestes dados, parece-nos que o primeiro passo da intervenção psicológica junto dos jovens mais propensos a desenvolver uma estrutura ansiosa será a construção de uma relação terapêutica de confiança e segurança. O psicólogo deve agir como uma base segura, sendo responsivo e disponível para criar confiança e apoiar o jovem para explorar e rever os modelos internos do self, das figuras de vinculação e dos outros (Colin, 1996). Somente através da compreensão e do apoio que esta relação proporciona é que o indivíduo poderá explorar os aspectos dolorosos do seu passado e do presente (Bowlby, 1989/95).

Com base nesta relação terapêutica de segurança e confiança, um segundo momento da intervenção será o de explorar a história de vinculação do sujeito. Esta exploração poderá basear-se em entrevistas que conduzam a memórias relativas aos seus modelos internos, em frases com conteúdos que apelem às recordações, ou recorrendo a filmes, fotos, escrita e às narrativas do sujeito acerca das suas experiências passadas. Esta exploração permitirá ao cliente perceber e reconstruir os modelos internos, reestrutu- 
rando significados das experiências vividas e integrá-los nas experiências actuais.

Um terceiro momento da intervenção será, então, centrado em estratégias que ajudem o jovem a tornar os seus modelos internos conscientes, de modo a percepcionarem de que forma contribuíram para a visão de si próprio e do mundo. No caso dos indivíduos com estilo de vinculação preocupado, esta tarefa pode ser facilitada por serem menos resistentes em tornar conscientes os seus modelos internos (Simpson \& Rholes, 1994), comparativamente aos outros padrões ansiosos (amedrontados e desinvestidos), provavelmente devido a terem menores experiências de rejeição e apresentarem um modelo dos outros positivo. O jovem deve consciencializar-se de que as suas percepções e expectativas presentes podem ser produto de acontecimentos e situações que enfrentou na sua infância e adolescência, na relação com os seus pais e que os seus modelos internos podem não estar ajustados ao presente (Bowlby, 1989/95). Assim, compreender a origem dos seus pensamentos, sentimentos e expectativas pode ajudar o jovem a compreender o modo como se vê a si próprio e como se relaciona com os outros.

Numa fase seguinte, a intervenção deve incidir na reconstrução de significados e emoções, de modo a construir um modelo interno de si próprio positivo, como ser amável, respeitável e competente. Esta, porém, não é uma tarefa fácil, porque os modelos internos são estruturas que contêm informação episódica, semântica e afectiva acerca das experiências com as figuras significativas e acerca dos acontecimentos interpessoais (Collins \& Read, 1994), cuja alteração implica mudanças profundas (Guidano \& Liotti, 1983). Assim, a intervenção psicológica deve promover a mudança destas estruturas representacionais tendo em consideração: (1) regras relativas ao tipo de emoções que devemos ou podemos ter numa relação; (2) modos de regulação do afecto; (3) crenças e valores associadas a si próprio e às relações com os outros (4) emoções dolorosas associadas às relações passadas e (5) expectativas acerca das relações futuras.

As mudanças nos modelos internos vão exigir ao jovem alterações ao nível do seu autoconceito, auto-imagem, auto-estima, implicando um trabalho de re-síntese e criação de novos significados acerca de si próprio, contribuindo para uma visão de si próprio e dos outros positiva e, portanto, mais confiante e segura. Esta nova visão de si próprio permitirá ao jovem aceitar os vários desafios psicossociais (tirar um curso, investir em novas relações, conhecer novos locais, desempenhar novas responsabilidades) de modo autónomo, sem medos (viajar, fobia social, agorafobia) e sem preocupações ou dúvidas excessivas (pensamentos e impulsos obsessivos, compulsões de verificação e limpeza) de ser rejeitado ou abandonado pelos 
outros, e sem viver de forma ansiosa o afastamento das suas figuras protectoras, geralmente os pais.

No entanto, ainda que a intervenção possa ter uma modalidade predominantemente individual, pode e, nalguns casos, deve realizar-se também ao nível do sistema familiar. A intervenção junto da família é importante, no sentido de promover uma comunicação clara que facilite o questionamento do jovem acerca dos significados que lhe são atribuídos (Matos, 2002), de desenvolver conflitos interpessoais que possam interferir com a segurança do jovem ou impedir o seu desenvolvimento. A intervenção dirigida à família pode ainda ser intencionalizada, de modo a promover a mudança de estilos educativos, por exemplo, de superproteção, e de facilitar a autonomia do jovem.

Do mesmo modo, a intervenção pode ser alargada à promoção de relações significativas com os pares, no sentido de ajudar e reforçar a reestruturação realizada em consulta. Estas relações podem funcionar como novos "espelhos" que devolvam uma imagem positiva ao jovem e sejam fonte de segurança.

Os momentos do processo de intervenção psicológica devem ser transversais ao longo da intervenção que, sendo orientada por uma perspectiva construtivo-desenvolvimental, deve ser ecléctica, podendo utilizar-se estratégias de outros modelos de intervenção.

Assim, sendo a juventude um momento de grandes mudanças pessoais e grandes desafios em termos interpessoais e sociais e, por isso, um momento da vida com maior vulnerabilidade, e atendendo a que esta fase implica um processo de revisão dos modelos de si próprio e dos outros (Matos, 2002) e exige uma re-síntese de todas as tarefas psicossociais anteriores (Costa, 1991), parece-nos ser um período oportuno e de grande relevância para a intervenção psicológica, no sentido de prevenir ou tratar a ansiedade.

\section{Reflexões finais}

Apesar do contributo que possamos retirar deste estudo, é necessário ter em consideração que se trata de um estudo exploratório que encerra algumas limitações. Com efeito, trata-se de uma amostra de estudantes universitários, pelo que não deve ser considerada representativa de toda a população jovem, devendo ter-se cuidado com generalizações destes resultados.

Será fundamental o alargamento a outras populações não estudantis, para permitir analisar a influência de outros contextos socioculturais, bem como o estudo comparativo com amostras clínicas. Seria também interessante ter em consideração outros factores, como a influência do género, dos pares, da família e ainda factores biológicos e culturais, na medida que 
são factores mencionados na literatura como sendo um risco ou protecção relativamente à ansiedade.

Outro aspecto igualmente relevante seria o recurso a metodologias de natureza longitudinal, no sentido de avaliar a evolução e transformações ocorridas ao longo do desenvolvimento.

Em conclusão, esperamos ter contribuído, apesar das limitações apontadas, para salientar a importância que a construção de uma ligação segura a pessoas significativas tem no desenvolvimento e expressão da ansiedade. A possibilidade que a teoria da vinculação dá de reconstrução da visão de si próprio, dos outros e do mundo, com base numa relação significativa, permite olhar a intervenção psicológica na juventude como um momento por excelência para a prevenção de estruturas ansiosas e para a promoção de um desenvolvimento psicológico saudável.

\section{Referências}

Allen, J. P., Moore, C., Kuperminc, G., \& Bell, K. (1998). Attachment and adolescent psychosocial functioning. Child Development, 69, 1406-1419.

Allen, J. P., \& Land, D. (1999). Attachment in adolescence. In J. Cassidy \& S. R. Shaver (eds.), Handbook of attachment: Theory and clinical applications (pp. 319-335). Nova Iorque: The Guilford Press.

Armsden, G. C., \& Greenberg, M. T. (1987). The inventory of parenting and peer attachment: Individual difference and their relationship to psychological well-being in adolescence. Journal of Youth and Adolescence, 16, 427-45.

Ainsworth, M. D., Blehar, M. C., \& Wall, S. (1989). Attachments beyond infancy. American Psychologist, 44, 709-716.

Barbosa, M. R., (2001). A vinculação aos pais e a imagem corporal de adolescentes e jovens. Tese de Mestrado não publicada. Porto: Faculdade de Psicologia e de Ciências da Educação da Universidade do Porto.

Bartholomew, K. (1990). Avoidance of intimacy: An attachment perspective. Journal of Social and Personal Relationship, 7, 147-178.

Bartholomew, K., \& Horowitz, L. (1991). Attachment styles among young adults: A test of four category model. Journal of Personality and Social Psychology, 61, 226-224.

Beidel, D. C., Turner, S. M., \& Morris, T. L. (1999). The psychopathology of childhood social phobia. Journal of the American Academy of Child and Adolescent Psychiatry, 38, 643-650.

Boer, F., \& Lindhout, I. (2001). Family and genetic influences: Is anxiety 'all in the family'? In W. K. Silverman \& P. D. A. Treffers (Eds.), Anxiety disorders in children and adolescents - Research, assessment and intervention (pp. 235-254). Reino Unido: Cambridge University Press.

Bretherton, I. (1985). Attachment theory: Retrospect and prospect. In I. Bretherton \& E. Waters (Eds.), Growing points of attachment theory and research. Mono- 
graphs of the Society for Research in Child Development, 50 (1-2, serial Nr. 129), 3-38.

Bowlby, J. (1982). Attachment and loss: Attachment. Londres: The Hogarth Press.

Bowlby, J. (1973). Attachment and loss: Separation, anxiety and anger. Nova Iorque: Basic Books.

Bowlby, J. (1979). The making and breaking of affectional bonds. Londres: Routledge.

Bowlby, J. (1980). Attachment and loss: Loss, sadness and depression. Nova Iorque: Basic Books.

Bowlby, J. (1989/95). Una base segura. Aplicaciones clínicas de una teoría del apego. Barcelona: Paidós Psicologia Profunda.

Canavarro, M. C. S. (1999). Relações afectivas e saúde mental - Uma abordagem ao longo do ciclo de vida. Coimbra: Quarteto Editora.

Cassidy, J., \& Berlin, L. J. (1994). The insecure/ambivalent pattern of attachment: Theory and research. Child Development, 65, 971-991.

Cassidy, J. (1995). Attachment and generalized anxiety disorder. In D. Cicchetti \& S. Toth (Eds.), Emotion, cognition, and representation: Rochester symposium on developmental psychopathology (vol. VI, pp. 343-70). Nova Iorque: University of Rochester Press.

Collins, N. L., \& Read, S. J. (1994). Cognitive representations of attachment: The structure and function of working models. In K. Bartholomew \& D. Perlman (Eds.), Attachment processes in adulthood (vol. 5, pp. 53-92). Londres: Jessica Kingsley Publ.

Colin, V. L. (1996). Human attachment. Nova Iorque: McGraw-Hill.

Costa, M. E. (s/d). Ansiedade e medo: Uma perspectiva desenvolvimental (policopiado não publicado). Porto: Faculdade de Psicologia e Ciências da Educação da Univerisdade do Porto.

Cowan, P. A., Cwan, C. P., Cohn, D. A., \& Pearson, J. L. (1996). Parents attachments histories and children's externalizing and internalizing behaviors: Exploring family systems models of linkage. Journal of Consulting and Clinical Psychology, 64, 53-63.

Crowell, J. A. (2003). Assessment of a attachment security in a clinical setting: Observations of parents and children. Journal of Developmental \& Behavioral Pediatrics, 24 (3), 190-204.

Cunha, M., \& Gouveia, J. P. (1999). Ansiedade social e auto-representação: contributo dos esquemas precoces mal-adaptativos e dos esquemas cognitivos interpessoais. Psychologica, 21, 5-25.

Dadds, M. R., \& Roth, J. H. (2001). Family process in the development of anxiety problems. In M. W. Vasey \& M. R. Dadds (Eds.), The developmental psychopathology of anxiety (pp. 278-303). Oxford: University Press.

Emmelkamp, P. M., Bouman, T. K., \& Scholing, A., (1992). Anxiety disorders. Nova Iorque: John Wiley \& Sons.

Erikson, E. H. (1968). Identidade, juventude e crise. Rio de Janeiro: Zahar Editores. 
Greenberg, M. T. (1999). Attachment and psychopathology in childhood. In J. Cassidy, \& P. R. Shaver (Eds.), Handbook of attachment: Theory research, and clinical applications (pp. 469-495). Nova Iorque: The Guilford Press.

Guidano, V. F., \& Liotti, G. (1983). Cognitive processes and emotional disorders. Nova Iorque: The Guildford Press.

Guidano, V. F. (1991). The Self in process: Toward a post-rationalist cognitive therapy. Nova Iorque: The Guildford Press.

Horowitz, L. M., Rosenbertg, S. E., \& Bartholomew, K. (1993). Interpersonal problems, attachment styles, and outcome in brief dynamic psychotherapy. Journal of Consulting and Clinical Psychology, 61, 549-560.

Joffe, R. T., Swinson, R. P., \& Regan, J. J. (1988). Personality features of obsessive-compulsive disorder. American Journal of Psychiatry, 145, 1127-1129.

Kobak, R. (1999). The emotional dynamics of disruptions in attachment relationships. In J. Cassidy \& S. R. Shaver (Eds.), Handbook of attachment: Theory, research and clinical applications (pp. 469-495). Nova Iorque: The Guilford Press.

Kochanska, G. (2001). Emotional development in children with different attachment histories: The first three years. Child Development, 72 (2), 474-490.

Landy, S. (2002). Pathways to competence - Encouraging healthy social and emotional development in young children. USA: Paul H. Publishing CO, Inc.

Lapsey, D. K., Rice, K., \& Fitzgerald, D. P. (1990). Adolescent attachment, identity and adjustment to college implications for the continuity of adaptation hypothesis. Journal of Counseling and Development, 68, 561-565.

Lonigan, C. J., \& Phillips, B. M. (2001). Temperamental influences on the development of anxiety disorders. In M. W. Vasey \& M. R. Dadds (Eds), The developmental psychopathology of anxiety (pp. 60-91). Nova Iorque: Oxford University Press.

Lyddon, W. J., \& Sherry, A. (2001). Developmental personality styles: An attachment theory conceptualization of personality disorders. Journal of Counseling and Development, 79, 405-414.

Manassis, K., Bradley, S., Goldberg, S., Hood, J., \& Swinson, R. P. (1994). Attachment in mothers with anxiety disorders and their children. Journal of the American Academy of Child and Adolescent Psychiatry, 33, 1106-1113.

Manassis, K. (2001). Child-parent relations: attachment and anxiety disorders. In W. K. Silverman \& P. D. A. Treffers (Eds), Anxiety disorders in children and adolescents - Research, assessment and intervention (pp. 255-272). Reino Unido: Cambridge University Press.

Marks, I., \& Mathews, A. (1979). Brief standard self-rating for patients. Behavior Research Therapy, 17, 263-267.

Marsh, P., McFarland, F. C., Allen, J. P., McElhaney, K. B., \& Land, D. (2003). Attachment, autonomy, and multifinality in adolescent internalizing and risky behavioral symptoms. Development and Psychopathology, 15, 451-467.

Matos, P. M., Almeida, H. M., \& Costa, M. E. (1997). O questionário de vinculação ao pai e à mãe: Desenvolvimento e estudos de validação (não publicado). 
Matos, P. M., Barbosa, S., Almeida, H. M., \& Costa, M. E. (2001). Avaliação da vinculação amorosa em adolescentes e jovens adultos: Construção de um instrumento e estudos de validação. RIDEP, 11 (1), 94-109.

Matos, P. M., Barbosa, S., Almeida, H. M., \& Costa, M. E. (1999). Attachment and identity in Portuguese late adolescents. Journal of Adolescence, 22, 805-818.

Matos, P. M. (2002). (Des)Continuidades na vinculação aos pais e ao par amoroso. Tese de doutoramento não publicada. Porto: Faculdade de Psicologia e de Ciências da Educação da Universidade do Porto.

Morris, T. (2001). Social phobia. In M. W. Vasey \& M. R. Dadds (Eds), The developmental psychopathology of anxiety (pp. 435-458). Nova Iorque: Oxford University Press.

Oosterlaan, J. (2001). Behavioural inhibition and the development of childhood anxiety disorders. In W. K. Silverman \& P. D. A. Treffers (Eds.), Anxiety disorders in children and adolescents - Research, assessment and intervention (pp. 45-71). Nova Iorque: Cambridge University Press.

Paterson, J., Pryor, J., \& Field, J. (1995). Adolescent attachment to parents and friends in relation to aspects of self-esteem. Journal of Youth and Adolescence, 24, 365-376.

Raja, S. N., McGee, R., \& Stanton, W. R. (1992). Perceived attachments to parents and peers psychological well-being in adolescence. Journal of Youth and Adolescence, 21, 471-485.

Routh, D., \& Bernholtz, J. (1991). Attachment, separation and phobias. In J. Gewirtz \& W. Kurtines (Eds.), Intersections with attachment (pp. 295-309). Nova Jérsia: Lawrence Erbaum Associates, Publishers.

Rutter, M. (1999). Clinical implications of attachment concepts: Retrospect and prospect. In J. Cassidy \& P. R. Shaver (Eds.), Handbook of attachment: Theory research, and clinical applications (pp. 497-518). Nova Iorque: The Guilford Press.

Salvador, M. C. T. (1997). Ansiedade e fobia social. In Processamento de informação na fobia social: O teste de Stroop modificado (pp. 8-105). Tese de Mestrado não publicada. Coimbra: Faculdade de Psicologia e de Ciências da Educação da Universidade de Coimbra.

Sanavio, E. (1988). Obsessions and compulsions: The Padua inventory. Behaviour Research and Therapy, 26 (2), 169-177.

Simpson, J. A., \& Rholes, W. S. (1994). Stress and secure base relathionship in adulthood. In K. Bartholomew \& D. Perlman (Eds.), Attachment processes in adulthood (vol. 5, pp. 181-204). Londres: Jessica Kingsley Publ.

Sroufe, L. A. (1990). Considering normal and abnormal together: The essence of developmental psychopathology. Development and Psychopathology, 2, 103$-113$.

Sroufe, L. A., Carlson, E. A., Levy A. K., \& Egeland, B. (1999). Implications of attachment theory for developmental psychopathology. Development and Psychopathology, 11, 1-13.

Steketee, G. S. (1993). Treatment of obsessive-compulsive disorder. Nova Iorque: The Guilford Press. 
Thompson, R. A. (2001). Childhood anxiety disorders from the perspective of emotion regulation and attachment. In M. W. Vasey \& M. R. Dadds (Eds.), The developmental psychopathology of anxiety (pp. 160-182). Oxford: University Press.

Warren, S. L., Huston, L. Egeland, B., \& Sroufe, L. A. (1997). Child and Adolescent anxiety disorders and early attachment. Journal of the American Academy of Child and Adolescent Psychiatry, 36, 637-644.

Wartner, U. G., Grossmann, K., Fremmer-Bombik, E., \& Suess, G. (1994). Attachment patterns at age six in south Germany: Predictability from infancy and implications for preschool behavior. Child Development, 65, 1014-1027.

Weinfield, N: S., Sroufe, L. A., Egeland, B., \& Carlson, E. A. (1999). The nature of individual differences in infant-caregiver attachment. In J. Cassidy \& S. R. Shaver (Eds.), Handbook of attachment: Theory, research and clinical applications (pp. 68-87). Nova Iorque: The Guildford Press.

Wolpe, J. \& Lazarus, A. A. (1966). Behaviour therapy technique. Oxford: Pergamon Press. 